\title{
A compact air cooling system for testing silicon detectors based on a vortex chiller
}

\author{
K.Metodiev $^{\mathrm{a}}$, L.Vigani ${ }^{\mathrm{a}}$, R.Plackett ${ }^{\mathrm{a}}$, K.Arndt ${ }^{\mathrm{a}}$, D.Wood ${ }^{\mathrm{a}}$, D.P.Weatherill ${ }^{\mathrm{a}}$, \\ M.Mironova $^{\mathrm{a}}$, D.Bortoletto ${ }^{\mathrm{a}}$, I.Shipsey ${ }^{\mathrm{a}}$ \\ ${ }^{a}$ University of Oxford, Department of Physics, Keble Road, Oxford, UK OX1 3RH
}

\begin{abstract}
The testing of irradiated silicon detectors requires maintaining low $\left(\leq-10{ }^{\circ} \mathrm{C}\right)$ temperatures, to simulate a realistic operating environment and prevent annealing effects from distorting the results of the measurement. Keeping a device cool and dry is challenging, particularly if the apparatus must be portable. This paper presents a solution for a providing a stable, cool and dry environment for testing an irradiated silicon detector, that is easy to transport and can be installed in charged particle beam areas and irradiation facilities.
\end{abstract}

Keywords: vortex, cooler, testbeam, dry, compressed

\section{Introduction}

In order to confirm that a silicon detector is suitably radiation hard for experiments operating at proton-proton colliders, it must be irradiated to a lifetime equivalent dose. Radiation damage degrades the performance of silicon devices, but this effect depends on the device and the magnitude and rate of the radiation field. The silicon bulk is affected by point like or cluster defects in the lattice, which then act as recombination centers to increase the leakage current, and as charge traps to reduce charge collection and alter the effective doping concentration, decreasing the depletion region [1]. Even after the irradiation is completed, its effects continue to evolve and affect a detector in processes referred to as annealing and anti-annealing [2]. Annealing is very temperature dependent, and as it is desirable to measure the actual effect of the irradiation on the device, the device is cooled to slow the annealing. Temperatures below $-10{ }^{\circ} \mathrm{C}$ are good enough for most small scale experiments that test the device. To prevent frost or moisture appearing on the DUT (Device Under Test), the surrounding atmosphere must also be dry, lowering the dew point.

There are various ways to achieve a cold, dry environment, including the use of dry ice [3], liquid nitrogen boil off, liquid chillers [4] and dry air combined with Peltier cooling [5]. Each of these methods suffers different drawbacks; the use of dry ice is inconvenient and unstable, whilst using Peltier cooling adds metal into the path of the beam and has a high power requirement for large $\Delta \mathrm{T}$. Liquid chillers most often require a Peltier cooler, custom parts for each 
device as well as thermal vias need to be present on the printed circuit board (PCB), in order to avoid metal on the beamline. Additionally most setups are not easily portable, and many test-beam locations are not equipped with all of the required apparatus, such as liquid nitrogen.

The cooling of irradiated devices in the lab and at test-beams using a cold air stream produced by a vortex cooler has been investigated [6]. This is a small, compact device that can maintain a stable temperature and requires only dry compressed air to operate. The basic working principle of a vortex cooler is that a compressed air flow creates a vortex guided by vanes, resulting in a large centrifugal force which separates the hot and the cold air molecules (Fig 1). With a suitable vortex channel, input pressure and airflow the vortex cooler is capable of producing a $>10 \mathrm{ls}^{-1}$ of air at $<-25{ }^{\circ} \mathrm{C}$. To prevent the build-up of ice crystals, the compressed air supply must have a dew point below the coldest temperature produced by the vortex cooler. As this combination of pressure, flow, and dryness is not always available at user facilities, a commercially available portable compressor with an attached air drying system was used (Hydrovane HV04 ${ }^{1}$ ).

The DUT was enclosed in a polystyrene cold-box specifically designed for the required devices to minimize the volume of air to be cooled and to ensure efficient cooling. Since pixel detector chips are small devices with a large surface area, the cold airflow alone is enough to keep them at desired temperature and provide a dry environment within the enclosure. The vortex cooler was first tested using a 'dummy' chip and wire bonds inside the lab, and then the entire setup was used to provide cooling for an irradiated sensor at the DESY II test beam facility [7]. Afterwards the vortex cooler was integrated in an edge transient-current technique (E-TCT)[8] setup for a device, which requires cooling without thermal contact. The following sections of the paper outline the results of these three sets of tests.

\section{Laboratory testing}

To confirm that the setup can reach the required temperatures, it was tested using a flat flex heater similar in size to a large pixel detector chip, through which various currents were passed to test the cooling performance across a range of dissipated power. The setup is shown in Fig 2.

A range of dissipated power between $0 \mathrm{~W}$ and $11 \mathrm{~W}$ was tested to the requirements of most high-performance pixel detector modules. It was observed that for a given power dissipation, the device temperature remains constant. Thus proving that the air stream is able to maintain an equilibrium at a cold temperature. The results are shown in Fig 3. It should be noted that it is

\footnotetext{
${ }^{1}$ Pressure can be up to 7 bar, flow can be up to $11 \mathrm{ls}^{-1}$ and it has a membrane dryer with a dew point at $-25{ }^{\circ} \mathrm{C}$. Its dimensions are $455 \times 1410 \times 900 \mathrm{~mm}$.
} 


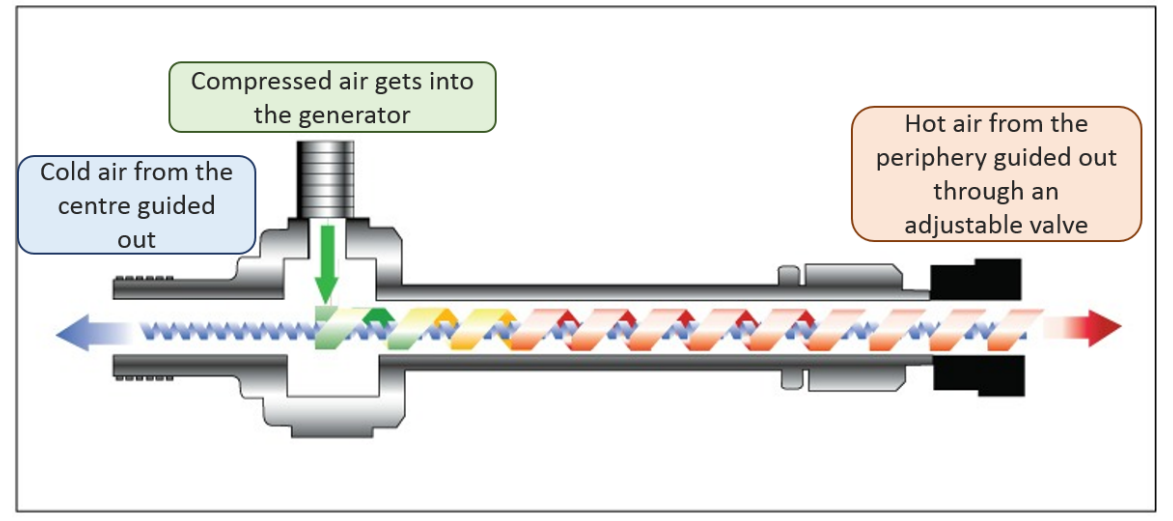

Figure 1: Sketch of the airflow inside the vortex cooler (adapted from [9]). The high pressure air coming in is guided to create a vortex, which results in a large centrifugal force that separates the hot air particles to the periphery of the inner pipe. The hot air is then released through an adjustable valve at one end of the cooler, while the cold air is guided out from the other side. (colour online)

important to insulate the tube carrying cold air from the vortex cooler to the inside of the box enclosing DUT, in order to minimize thermal losses. The temperature at equilibrium is dependent on these losses, as well as on any leaks in the box enclosing the DUT. For these tests we found that it was sufficient to seal any leaks in the enclosure with Kapton tape.

To evaluate the potential wire bond damage from the airflow of the vortex cooler, test wire bonds were made. They were exposed for two hours directly and as close as possible to the cold end of the vortex cooler, when the cooler was supplied with 6 bar of dry air with airflow measured at $2.3 \mathrm{ls}^{-1}$. Although the system does not produce a steep pressure gradient due to its valves, it was additionally turned on and off multiple times to try and produce a shock on the wire bonds. These conditions would give the highest likelihood for wire bond damage, but no harmful effect of the high airflow and pressure gradient was observed (Fig 4).

\section{Performance at a test-beam}

The complete apparatus, consisting of cooler, compressor and polystyrene enclosure was transported to the DESY test-beam facility (Fig 5) as part of a program of tests [10] of an irradiated LFCPIX HVCMOS depleted MAPS device [11]. The only requirement from the facility was a three-phase power supply to run the air compressor.

Due to the size of the DUT, and the presence of the readout boards and cables, some leaks in the encapsulation box were inevitable, meaning that a slightly 

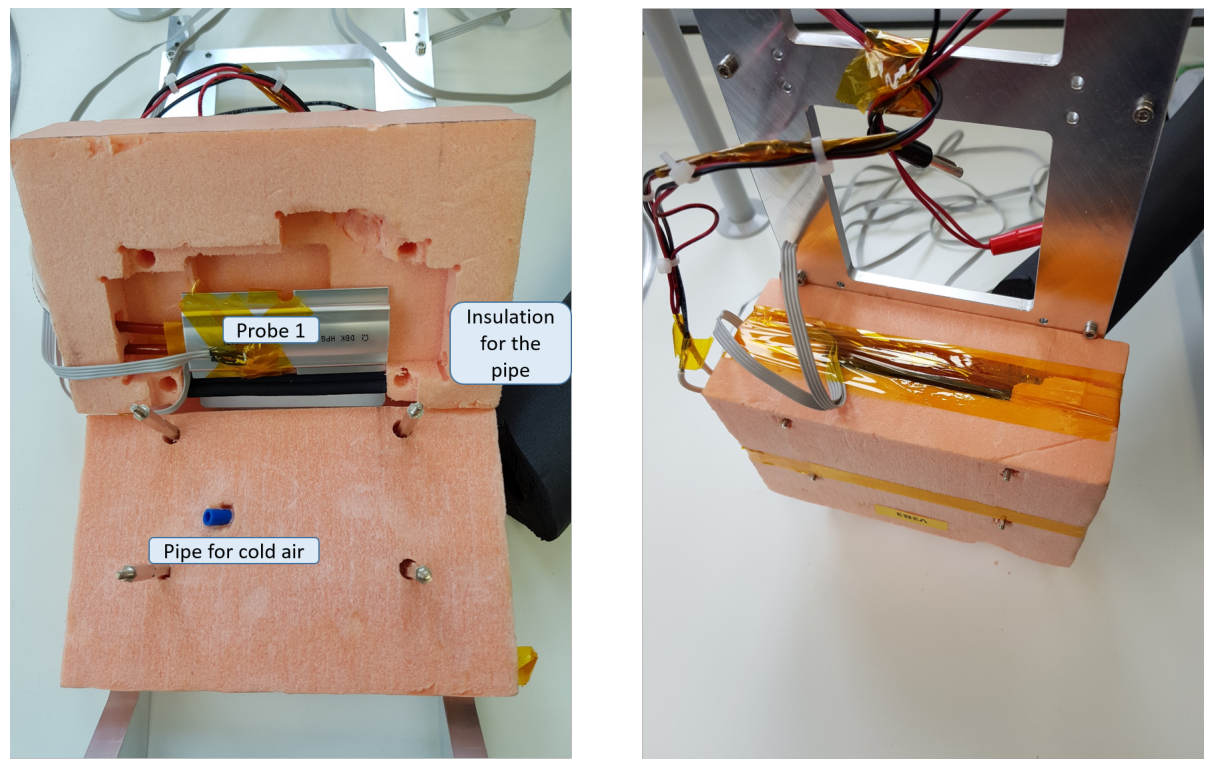

Figure 2: The photo on the left-hand side shows the setup used in the lab to measure the temperature at different values of power dissipation from the heater. Temperature probe 1 is taped to the silver-coloured flex heater while probe 2 is behind the heater. The photo on the right-hand side shows the closed box with the holes sealed using Kapton tape.

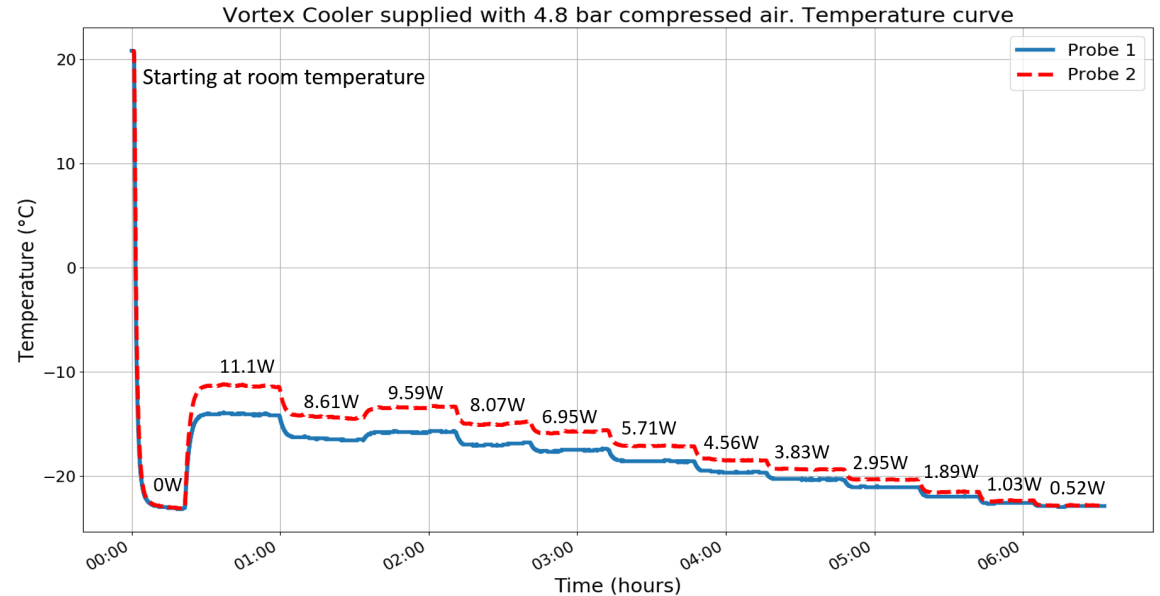

Figure 3: Readings of 2 temperature sensors mounted on the two sides of the flex heater. The pressure of the incoming compressed air is set to 4.8 bars and as the minimum temperature is reached, the device is turned on and kept at each power value for $\sim 30$ minutes. Probe 1 is directly exposed to the cold air while Probe 2 is located behind the flex heater. 
(a)

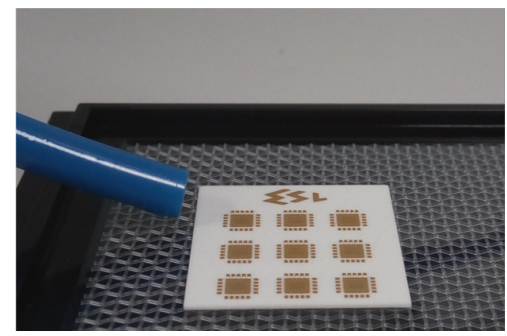

(b)

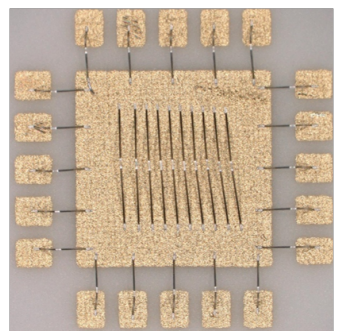

(c)

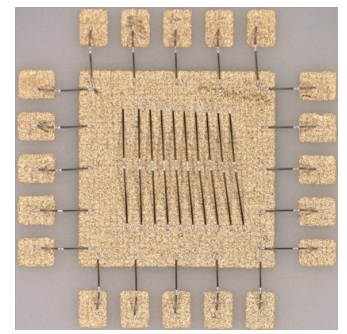

Figure 4: (a) Photo of the test wire bonds being exposed to the air flow coming out of the vortex cooler. Photos under a microscope of the test wire bonds before (b) and after (c) being exposed to the high airflow are shown.

greater air pressure compared to the lab conditions was required for comparable levels of cooling. The holes that were used for the electrical connections required removing the frost build-up. For this purpose the room temperature dry compressed air from the warmer end of the vortex cooler was channeled using four small pipes to all parts of the junction with the readout board, that needed to be kept dry.

An unexpected effect that was encountered was the occasional build-up of ice in the cold air transmission pipe; this was due to much colder than expected temperatures being produced at the 'tip' of the vortex. This led to the formation of ice crystals even though the supplied dry air had a dew point of below $-30{ }^{\circ} \mathrm{C}$. These crystals would occasionally stick at joints or valleys along the pipe, and build up to form a blockage that would disrupt the airflow and hence the cooling. This issue was addressed by running the system below maximum pressure and flow, and therefore avoiding very cold temperatures and maintaining a negative spatial gradient of the pipe. The optimised setup sustained a temperature of $-17.5^{\circ} \mathrm{C}$ for the 36 hours of testing with a device dissipating an average power of $0.85 \mathrm{~W}$ as shown in Fig 6 .

\section{Cooling in an E-TCT setup}

The Edge Transient-Current Technique (E-TCT) is a powerful method for measuring the depletion region of a sensor by using an infrared laser to scan the silicon in its depth [8]. The vortex cooling system was integrated in our E-TCT setup during the testing of the TowerJazz Investigator 1 chip [12]. This is a prototype test chip developed for evaluating the silicon bulk characteristics of a potential depleted monolithic active pixel sensor for ATLAS ITk [13].

The PCB carrier of the chip does not have a thermal connection to the back of the chip. Therefore cooling of the air around the chip was needed. The cold end of the vortex cooler was pointed as close as possible to the back of the chip and a polystyrene enclosure was built around the motion stages and the DUT (Fig 7). Due to the inevitable leaks and large volume (18 l) of the enclosure, 

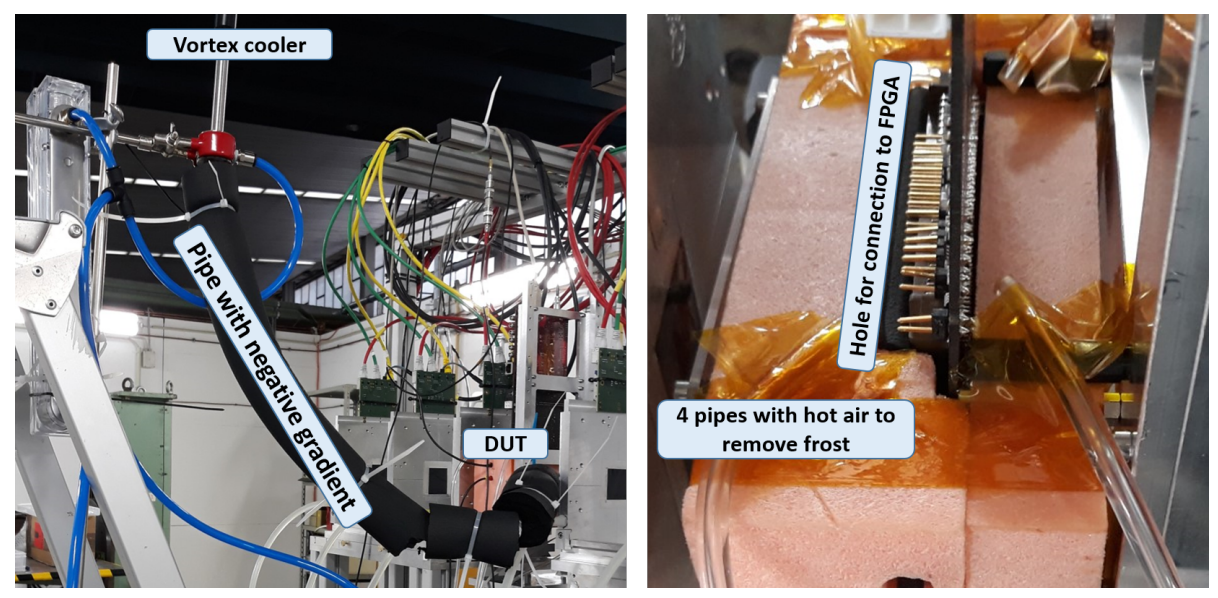

Figure 5: Photos of the setup at the test-beam facility. The vortex cooler is located above the DUT to maintain a constant negative gradient of the airflow (left). The 4 pipes blowing hot air on the largest gap in the box containing the DUT to prevent frost are shown on the right-hand side photo.

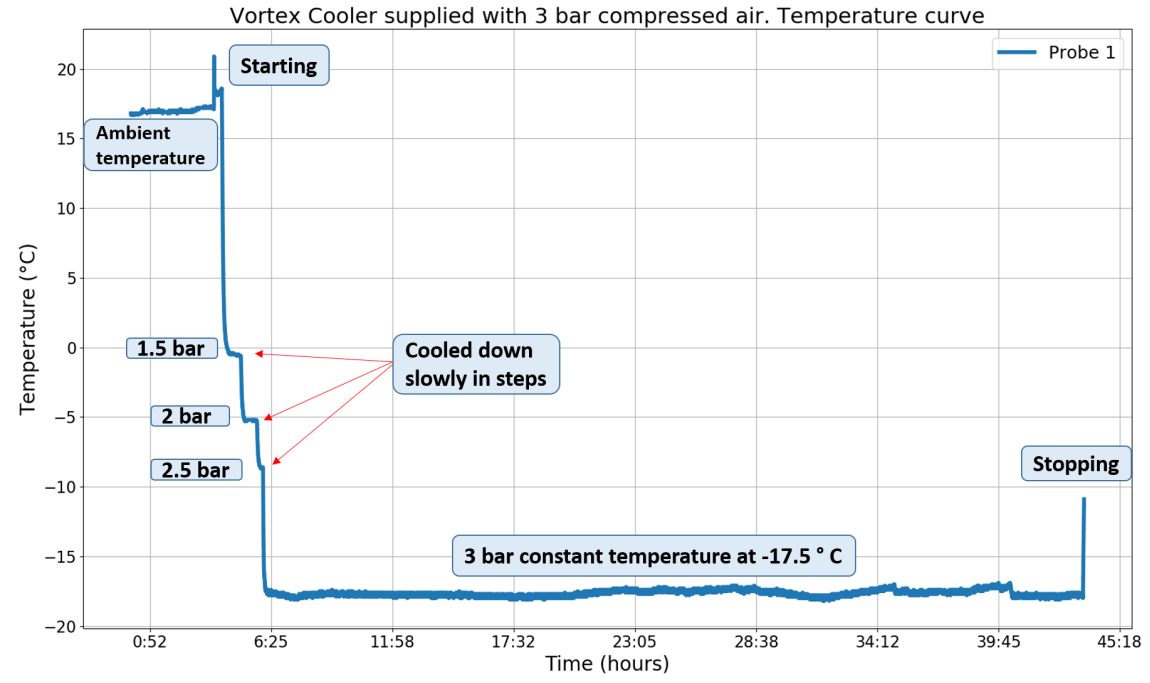

Figure 6: Temperature monitoring for one run at the test-beam facility. Probe 1 is exposed directly to the incoming air, equivalent to Probe 1 in the setup used for Fig 3. 

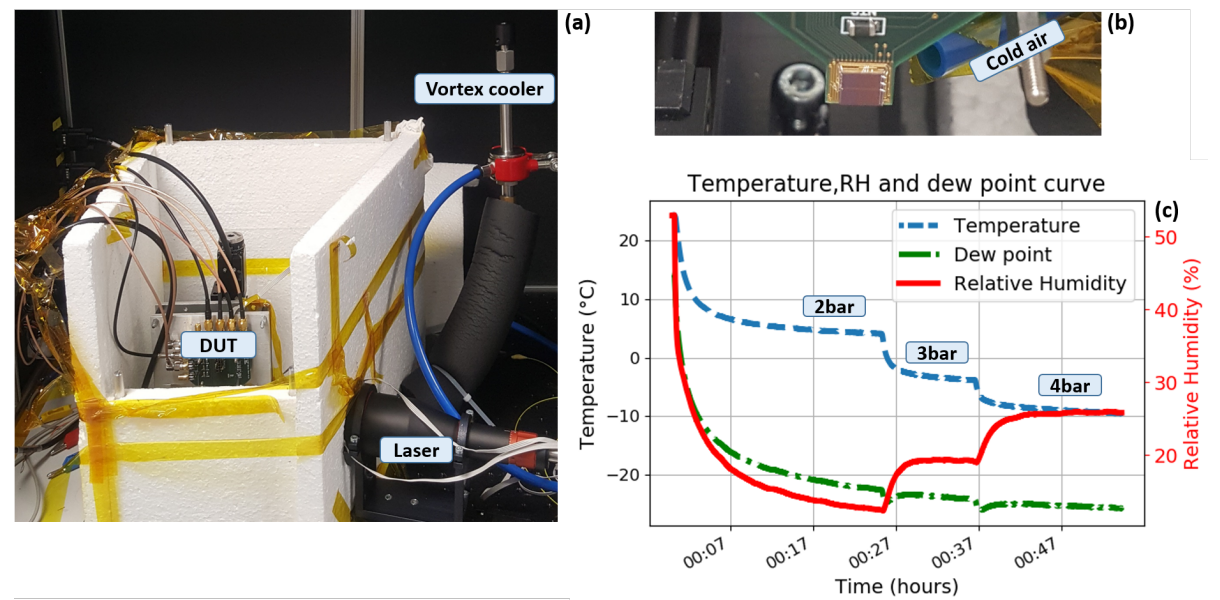

Figure 7: (a) A photo of the E-TCT setup with the polystyrene enclosure with the top off.(b) A close-up of photo of the DUT, showing the cold air flow coming to the back of the chip. (c) Temperature, relative humidity measurements at the back of the PCB, showing the environment characteristics around the chip. The dew point was estimated [14] for each combination of RH and temperature to make sure there is no condensation.

relative humidity and temperature sensors were placed on the PCB to monitor the properties of the environment around the chip (Fig 7(c)).

With maintaining an environment around the chip at $-10{ }^{\circ} \mathrm{C}$ and a dew point at least $10^{\circ} \mathrm{C}$ lower, the depletion region of the device was measured pre and post irradiation and a comparison was made (Fig 8). The studies showed that after a neutron equivalent fluence of $10^{15} \mathrm{~cm}^{-2}$, the depletion depth decreased from $26 \mu \mathrm{m}$ to $21 \mu \mathrm{m}$, which was still acceptable.

\section{Conclusions and future developments}

A vortex cooler, which is widely used in industry, low cost (400£) and portable has been shown to be an ideal solution for cooling silicon devices. The system is capable of providing and maintaining a stable cool, dry environment for the testing of silicon pixel detector chips, and is portable for uses at test-beams and other facilities. It can also be easily integrated and used for E-TCT measurements.

The principal drawbacks of the system are the high airflow requirements $\left(>1.5 \mathrm{ls}^{-1}\right)$, the production of frost when using the portable air compressor, and the noise produced by the warm air exhaust when operating in lab conditions. A higher performance source of compressed air, which should be available at most facilities, is expected to eliminate the formation of frost observed at DESY. In fact with the high-performance compressor installed in the laboratory, which has a dew point of $-60{ }^{\circ} \mathrm{C}$, there was no evidence of frost. Investigations are ongoing to address the acoustic noise problem by either mounting a 'silencer' on 

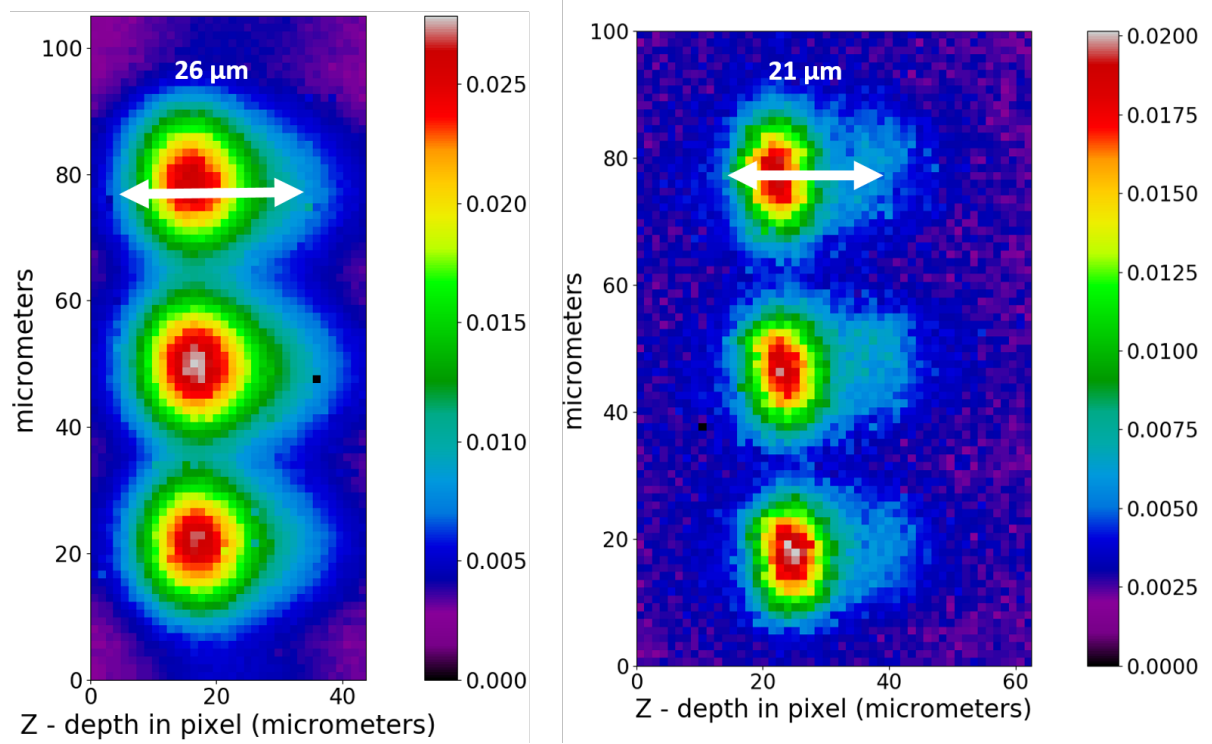

Figure 8: E-TCT scan result of 3 pixels from mini-matrix 106 of the Investigator 1 chip. The $\mathrm{x}$-axis shows the depletion depth (commonly denoted as Z). Calculation of the FWHM along the depth at the centre of the pixel was done to estimate the results shown for both the non-irradiated (left) and the irradiated (right) case.

the warm air exhaust or an additional pipe to guide the air outside, to make the system more compatible for lab use. This is slightly complicated by the need to allow the warm air to escape very freely to avoid interfering with the operation of the vortex cooler.

\section{References}

[1] G. Lindström, Radiation damage in silicon detectors, Nuclear Instruments and Methods in Physics Research Section A: Accelerators, Spectrometers, Detectors and Associated Equipment 512 (1) (2003) 30 - 43, ISSN 0168-9002, doi:https://doi.org/10.1016/S0168-9002(03)01874-6, URL http://www.sciencedirect.com/science/article/pii/S0168900203018746, proceedings of the 9th European Symposium on Semiconductor Detectors: New Developments on Radiation Detectors.

[2] Z. Li, Radiation damage effects in Si materials and detectors and rad-hard Si detectors for SLHC, Journal of Instrumentation 4 (3), ISSN 17480221, doi:10.1088/1748-0221/4/03/P03011.

[3] P. Prophys, Testbeam Measurements with Pixel Sensors for the ATLAS Insertable b-Layer Project . 
[4] M. Benoit, S. Braccini, G. Casse, H. Chen, K. Chen, F. A. Bello, D. Ferrere, T. Golling, S. Gonzalez-Sevilla, G. Iacobucci, M. Kiehn, F. Lanni, H. Liu, L. Meng, C. Merlassino, A. Miucci, D. Muenstermann, M. Nessi, H. Okawa, I. Perić, M. Rimoldi, B. Ristić, M. V. B. Pinto, J. Vossebeld, M. Weber, T. Weston, W. Wu, L. Xu, E. Zaffaroni, Testbeam results of irradiated ams H18 HV-CMOS pixel sensor prototypes, Journal of Instrumentation 13 (2), ISSN 17480221, doi:10.1088/1748-0221/13/02/P02011.

[5] A. Sokolov, V. Gostilo, A. Loupilov, V. Zalinkevich, Performance Improvement of $\mathrm{Si}(\mathrm{Li})$ Peltier Cooled Detectors, IEEE Transactions on Nuclear Science 49 (5) (2002) 3-6.

[6] Y. Xue, M. Arjomandi, R. Kelso, The working principle of a vortex tube, International Journal of Refrigeration 36 (6) (2013) 1730-1740, ISSN 01407007, doi:10.1016/j.ijrefrig.2013.04.016, URL http://dx.doi.org/10.1016/j.ijrefrig.2013.04.016.

[7] R. Diener, J. Dreyling-Eschweiler, H. Ehrlichmann, I. Gregor, U. Kötz, U. Krämer, N. Meyners, N. Potylitsina-Kube, A. Schütz, P. Schütze, M. Stanitzki, The DESY II test beam facility, Nuclear Instruments and Methods in Physics Research Section A: Accelerators, Spectrometers, Detectors and Associated Equipment 922 (2019) 265 - 286, ISSN 0168-9002, doi:https://doi.org/10.1016/j.nima.2018.11.133, URL http://www.sciencedirect.com/science/article/pii/S0168900218317868.

[8] A. Gorišek, V. Cindro, G. Kramberger, I. Mandić, M. Mikuž, M. Muškinja, M. Zavrtanik, The edge transient-current technique (E-TCT) with high energy hadron beam, Nuclear Instruments and Methods in Physics Research Section A: Accelerators, Spectrometers, Detectors and Associated Equipment 831 (2016) 250 - 253, ISSN 0168-9002, doi:https://doi.org/10.1016/j.nima.2016.03.061, URL http://www.sciencedirect.com/science/article/pii/S0168900216300845, proceedings of the 10th International "Hiroshima" Symposium on the Development and Application of Semiconductor Tracking Detectors.

[9] Air_Cooler_Bill.Pdf, URL http://aircoolernintama.blogspot.com /2017/02/vortex-air-cooler.html, online; accessed 02 October, 2018.

[10] L. Vigani, Study of CMOS prototypes for the ATLAS detector upgrade and searches for invisible decays of the Higgs boson, Ph.D. thesis, University of Oxford, 2018.

[11] L. Vigani, D. Bortoletto, L. Ambroz, R. Plackett, T. Hemperek, P. Rymaszewski, T. Wang, H. Krueger, T. Hirono, I. C. Sierra, N. Wermes, M. Barbero, S. Bhat, P. Breugnon, Z. Chen, S. Godiot, P. Pangaud, A. Rozanov, Study of prototypes of LFoundry active CMOS pixels sensors for the ATLAS detector, Journal of Instrumentation 13 (02) (2018) C02021, URL http://stacks.iop.org/1748-0221/13/i=02/a=C02021. 
[12] H. Pernegger, R. Bates, C. Buttar, M. Dalla, J. van Hoorne, T. Kugathasan, D. Maneuski, L. Musa, P. Riedler, C. Riegel, C. Sbarra, D. Schaefer, E. Schioppa, W. Snoeys, First tests of a novel radiation hard CMOS sensor process for Depleted Monolithic Active Pixel Sensors, Journal of Instrumentation 12 (06) (2017) P06008-P06008, doi:10.1088/17480221/12/06/p06008, URL https://doi.org/10.1088.

[13] B. Smart, ATLAS pixel detector design for the HL-LHC, Journal of Instrumentation 12 (02) (2017) C02011-C02011, doi:10.1088/17480221/12/02/c02011.

[14] H. E. Limodehi, M. Mozafari, H. Amiri, F. Légaré, Multi-channel fiber optic dew and humidity sensor, Optical Fiber Technology 41 (2018) 89 94, ISSN 1068-5200, doi:https://doi.org/10.1016/j.yofte.2018.01.006, URL http://www.sciencedirect.com/science/article/pii/S1068520017304716. 